\title{
Enhanced material defect imaging with a radio-frequency atomic magnetometer
}

\author{
Cite as: J. Appl. Phys. 125, 094503 (2019); doi: 10.1063/1.5083039 \\ Submitted: 27 November 2018 . Accepted: 12 February 2019 . \\ Published Online: 1 March 2019
}

P. Bevington, ${ }^{1,2}$ (D) R. Gartman, ${ }^{7}$ and W. Chalupczak ${ }^{1}$ iD

\author{
AFFILIATIONS \\ ${ }^{1}$ National Physical Laboratory, Hampton Road, Teddington TW11 OLW, United Kingdom \\ ${ }^{2}$ Department of Physics, University of Strathclyde, Glasgow G4 ONG, United Kingdom
}

\begin{abstract}
Imaging of structural defects in a material can be realized with a radio-frequency atomic magnetometer by monitoring the material's response to a radio-frequency excitation field. We demonstrate two measurement configurations that enable the increase of the amplitude and phase contrast in images that represent a structural defect in electrically conductive and magnetically permeable samples. Both concepts involve the elimination of the excitation field component, orthogonal to the sample surface, from the atomic magnetometer signal. The first method relies on the implementation of a set of coils that directly compensates the excitation field component in the magnetometer signal. The second takes advantage of the fact that the radio-frequency magnetometer is not sensitive to the magnetic field oscillating along one of its axes. Results from simple modelling confirm the experimental observation and are discussed in detail.
\end{abstract}

Published under license by AIP Publishing. https://doi.org/10.1063/1.5083039

\section{INTRODUCTION}

Implementation of radio-frequency (rf) magnetic fields in non-destructive testing provides a cost-effective option for the detection of structural defects, particularly in cases when there is no direct access to the surface of the studied sample. The technique relies on monitoring the material response to the so-called primary magnetic field $(\vec{B})$ created by an $\mathrm{rf}_{\text {coil. }}{ }^{1}$ The material response can be detected in a variety of ways. Traditionally, this is achieved by monitoring the impedance of the rf coil (or a dedicated pickup coil). ${ }^{1-5}$ However, the simplicity of instrumentation in this type of measurement is outweighed by the signal sensitivity degradation at low frequencies. Alternatives involve the implementation of magnetic sensors such as giant magnetoresistance (GMR) magnetometers, ${ }^{6-8}$ superconducting quantum interference devices (SQUIDs), ${ }^{9,10}$ and radio-frequency magnetometers. ${ }^{11-14,18}$ The magnetic field sensors directly monitor the response of the medium, the so-called secondary magnetic field $(\vec{b})$. The secondary field is produced by the primary magnetic field through eddy currents excited in electrically conductive samples, or magnetisation induced in samples with a magnetic permeability, ${ }^{18}$ and contains signatures of the inhomogeneities/structural defects within the sample.

The focus of our studies is material defect imaging with a $\mathrm{rf}$ atomic magnetometer. With superior field sensitivity $\left(\sim 1 \mathrm{fT} / \mathrm{Hz}^{1 / 2}\right),{ }^{15}$ better spatial resolution ${ }^{8}$ over standard pickup coils, the ability of tuning across a wide range of the operating frequencies ${ }^{16}$ that enables a change of the field penetration depth and the possibility of semi-vector field mapping ${ }^{17}$ makes the atomic magnetometer an attractive option for inductive measurements. We have previously demonstrated detection of defects (in the form of a recess) in carbon steel objects ${ }^{18}$ and analysed the shape of the spatial profiles observed in amplitude and phase images. ${ }^{17}$ The images recorded are produced from the variations in the phase and amplitude of the rf spectroscopy signal measured by the atomic magnetometer while scanning across the material. The rf field monitored by the magnetometer contains contributions from the primary and secondary magnetic field. A strong primary field contribution in the magnetometer signal results in the mapping of the two orthogonal components of the secondary field, $b_{z}$ and $b_{y}$, onto the amplitude and phase of the $\mathrm{rf}$ spectroscopy signal, respectively. We have previously reported that the component of the secondary field, $b_{y}$, parallel to the sample surface changes its sign in the vicinity of the surface edge (recess). ${ }^{17}$ As a consequence of a strong primary field, variations in the resultant field recorded by the rf atomic magnetometer measure the direction flip of the secondary field component; however, the observed rf signal phase change is smaller than the actual change in $b_{y}$.

In this paper, we demonstrate two simple methods of improving structural defect imaging with an $\mathrm{rf}$ atomic magnetometer. Both concepts involve the elimination of the primary field component, orthogonal to the sample surface, from the atomic 
magnetometer signal. The first relies on the implementation of a set of coils that creates an oscillating magnetic field, $\overrightarrow{B_{C}}$, that compensates the primary field contribution to the resultant field monitored by the atomic magnetometer vapour cell (Fig. 1), without changing the efficiency of the $\vec{b}$ excitation. The second method benefits from the fact that the rf atomic magnetometer is not sensitive to magnetic fields oscillating along the static bias field axis, $\vec{B}_{\text {bias }}$ (Fig. 1). When $\vec{B}_{\text {bias }}$ is oriented along $\vec{B}, B_{z}$ will be absent from the rf atomic magnetometer signal and the measurement configuration becomes equivalent to that when the primary field component is compensated. We are going to show that the elimination of the primary field component from the magnetometer signal significantly increases the phase and amplitude contrast of the images. This could provide a significantly quicker indication of a defect in non-destructive tests of large area samples. The concepts will be explored in the context of electrically conductive (aluminium) and magnetically permeable samples (carbon steel).

\section{EXPERIMENTAL SETUP}

Since the experimental setup is similar to that described in Refs. 17-19, only the essential components will be briefly discussed here. The measurement signal comes from the phase and amplitude change in the $\mathrm{rf}$ resonance spectra registered by an $\mathrm{rf}$ atomic magnetometer as a sample is moved under the rf coil (Fig. 1). The rf coil producing $\vec{B}$ is driven by the internally referenced output of a lock-in amplifier (SRS 865). The samples are fixed to a $2 \mathrm{D}$, computer-controlled translation stage. The sample is located approximately $30 \mathrm{~cm}$ from the cell and the coil is placed $1 \mathrm{~mm}-2$ $\mathrm{mm}$ above the sample surface. A set of two rf coils oriented along $\hat{z}$ and $\hat{y}$ creates an oscillating magnetic field, $\overrightarrow{B_{C}}$, that compensates the primary field seen by the atomic magnetometer. The

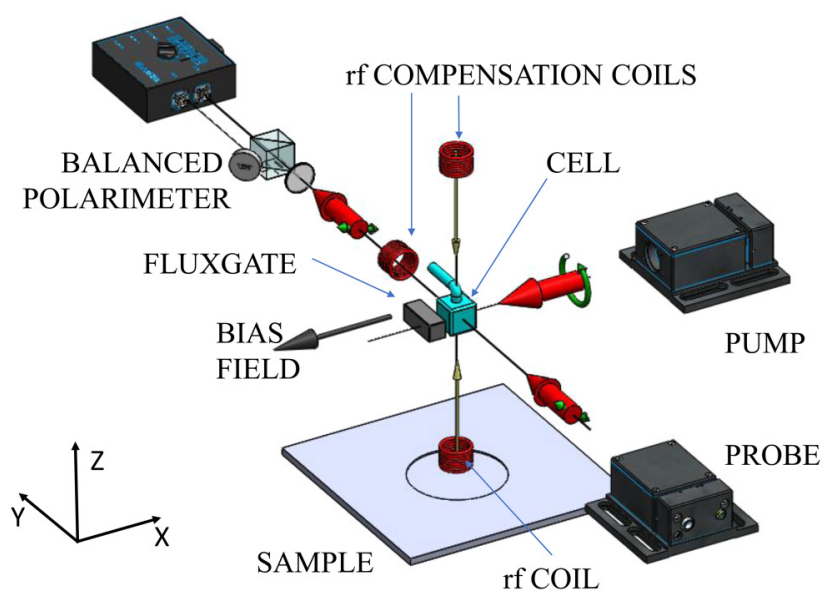

FIG. 1. Main components of the experimental setup. The secondary magnetic field is produced by the sample (plate with recess) response to the primary field created by the rf coil. The atomic magnetometer signal combines components created by the primary field and the secondary magnetic field. The primary field contribution is compensated in the vapour cell by a set of two if coils oriented along the $\hat{z}$ and $\hat{y}$ directions. compensation coils are driven by the output of the same lock-in amplifier used to generate $\vec{B}$. This keeps a constant phase difference between the fields $\vec{B}$ and $\overrightarrow{B_{C}}$. The dimensions of the coils producing the primary and compensation fields are the same (1000 turns of $0.2 \mathrm{~mm}$ diameter copper wire, height $10 \mathrm{~mm}$, and $2 \mathrm{~mm}$ and $4 \mathrm{~mm}$ inner and outer diameters). The amplitudes of the two components of $\overrightarrow{B_{C}}$ are varied by changing the distance of the respective coils from the vapour cell. The resultant magnetic field monitored by the rf atomic magnetometer includes components from the primary, secondary, and compensation field, i.e., $\vec{b}+\vec{B}+\overrightarrow{B_{C}}=$ $\vec{b}+\overrightarrow{B^{\prime}}$. The measurements are performed in a magnetically unshielded environment, where $\vec{B}_{\text {bias }}$ is stabilised by three pairs of nested, orthogonal, square Helmholtz coils. ${ }^{14,18,20}$ The strength of the bias field $\left(\left|\vec{B}_{\text {bias }}\right|\right)$ defines the operating frequency of the system (in most of the measurements equal to $12.6 \mathrm{kHz}$ ), i.e., the frequency of the magnetic resonance and the required primary field frequency. The atomic magnetometer is based on room temperature caesium atomic vapour (atomic density $n_{\mathrm{Cs}}=3.3 \times 10^{10} \mathrm{~cm}^{-3}$ ) housed in a paraffin-coated glass cell $\left(12 \times 12 \times 12 \mathrm{~mm}^{3}\right)$. Caesium atoms are optically pumped into the stretched state $\left(F=4, m_{F}=4\right)$ with a circularly polarised laser locked to the Cs $6^{2} \mathrm{~S}_{1 / 2} \mathrm{~F}=3 \rightarrow 6{ }^{2} \mathrm{P}_{3 / 2} \mathrm{~F}^{\prime}=2$ transition (D2 line, $852 \mathrm{~nm}$ ) propagating along the bias magnetic field $\vec{B}_{\text {bias }}$. The probe beam $(30 \mu \mathrm{W})$ is $580 \mathrm{MHz}$ blue shifted from the $6^{2} \mathrm{~S}_{1 / 2} \mathrm{~F}=4 \rightarrow 6{ }^{2} \mathrm{P}_{3 / 2} \mathrm{~F}^{\prime}=5$ transition via phase-offset-locking to the pump beam. Coherent spin precession of the Cs atoms is coupled to the polarisation of the probe beam (Faraday rotation) and is detected with a balanced polarimeter. Our experimental configuration, Fig. 1, allows the determination of amplitude and phase (orientation) of the rf field in the $y z$ plane, since only the components perpendicular to the bias magnetic field excite atomic precession. The amplitude $\left[R=\sqrt{\left(b_{y}+B_{y}^{\prime}\right)^{2}+\left(b_{z}+B_{z}^{\prime}\right)^{2}}\right]$ and the phase $\left(\phi=\arctan \frac{b_{y}+B_{y}^{\prime}}{b_{z}+B_{z}^{\prime}}\right)$ of the rf spectroscopy signal describe the changes in the resultant field, where $b_{z}+B_{z}^{\prime}$ and $b_{y}+B_{y}^{\prime}$ are the two quadrature components of the rf signal.

In most of the measurements, the frequency of the primary field has been scanned across the rf resonance, i.e., the whole resonance profile has been recorded, for each point of the image. ${ }^{18}$ This is a consequence of the imperfect field stabilisation system that compensates the change in the bias magnetic field as the ferromagnetic sample is being moved below the sensor ${ }^{17}$ and results in relatively long image acquisition time (around $12 \mathrm{~h}$ for $70 \times 70$ pixels image). We also tested an alternative mode of data acquisition, which significantly decreases the image acquisition time. In this mode, the modulation of the $\vec{B}$ frequency has been replaced with low-frequency modulation $(1 \mathrm{~Hz}-20 \mathrm{~Hz})$ of the amplitude of $\vec{B}_{\text {bias }}$. Since the amplitude of the bias field is stabilised with the fluxgate magnetometer, ${ }^{18}$ a small planar coil placed on top of the fluxgate magnetometer is used to modulate $\vec{B}_{\text {bias }}$. As a result of this, the output of the fluxgate contains an oscillatory component in the relevant direction. In this case, the signal recorded by a lock-in amplifier demodulating the magnetometer signal at the primary field frequency will contain a low-frequency oscillation with an amplitude equal to that of the rf resonance amplitude. A second lock-in amplifier referenced to the frequency of the $\vec{B}_{\text {bias }}$ amplitude modulation enables the readout of the rf resonance amplitude. The 
extent of the $\vec{B}_{\text {bias }}$ amplitude modulation can accommodate any imperfection in the bias field stabilisation, i.e., possible shifts in resonance frequency at different locations across the sample.

\section{MODELLING}

We use a simple 2D model based on Faraday's law to calculate the coupling between the primary field $\vec{B}$ and the conductive sample containing an inhomogeneity. ${ }^{17}$ We model the spatial distribution of $\vec{B}$ with a step function that describes the rf coil diameter. The secondary field, $\vec{b}$, changes linearly within the boundaries of the step function and decreases inversely with the distance outside of it. The eddy currents form closed loops that follow the path of least resistance. We begin with a model of $\vec{b}$ in the case of a $1 \mathrm{D}$ scan of the $\mathrm{rf}$ coil position in the $\hat{y}$ direction across the centre of the recess for an electrically conductive sample (Fig. 1; similar arguments could be used for objects with a magnetic permeability). In this geometry, only the $b_{y}$ and $b_{z}$ components have a non-zero value. Figure 2 shows the dependence of the secondary rf field components on the rf coil position. In a homogeneous sample (i.e., far from the recess), the eddy current flow has a circular symmetry and $\vec{b}$ has only one non-zero component, $b_{z}$ (solid blue line in Fig. 2). In the vicinity of the recess, the symmetry of the eddy current flow is broken and a non-zero component of $\vec{b}$ is produced in the $x y$ plane (dashed red line in Fig. 2). The asymmetry of the eddy current flow is mirrored on the other side of the recess, resulting in the opposite sign of $b_{y}$. In the case where the rf coil is above the recess, the magnetic flux through the plate becomes smaller since the coil lift-off (i.e., a distance between the $\mathrm{rf}$ coil and the plate surface) is greater, hence $|\vec{b}|$ decreases. We will refer to the maximum value of $b_{y}$ and $b_{z}$ within the recess boundaries as $b_{\mathrm{y} \text {,max }}$ and $b_{z, \max }$.

As we have shown previously, ${ }^{17}$ in the presence of a strong primary field $\vec{B}$ along $\hat{z}$, changes in the secondary field components, $b_{z}$ and $b_{y}$, are mapped onto the amplitude and phase of the rf spectroscopy signal, respectively. Changes in the resultant magnetic field monitored by the atomic magnetometer are relatively

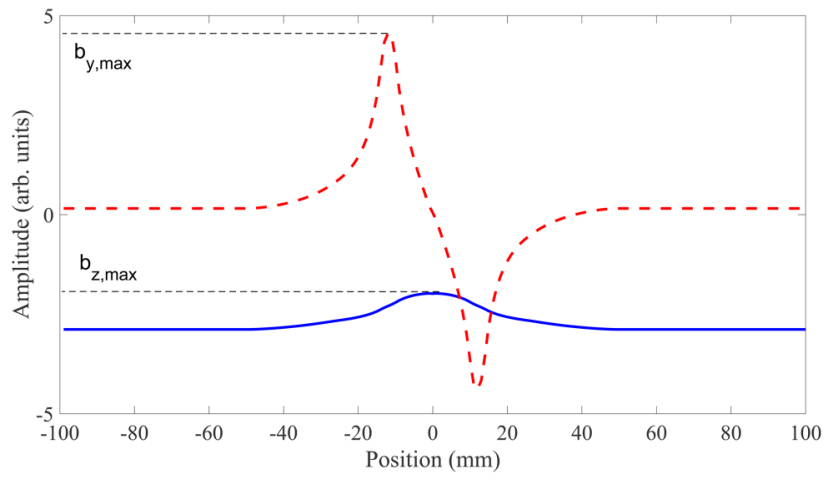

FIG. 2. Simulation of the secondary field components $b_{y}$ and $b_{z}$ (marked with dashed red and solid blue lines, respectively). The coil is moved along the $y$ axis across the centre of the recess. Maximum values of $b_{y}$ and $b_{z}$ within the recess boundaries are referred to as $b_{y, \max }$, and $b_{z, \max }$. small as they appear on top of a much bigger primary field. To provide better insight, we consider the case where $|\vec{b}| \sim \frac{1}{3}|\vec{B}|$ along both $\hat{z}$ and $\hat{y}$. Here, the observed amplitude and phase contrasts are estimated to be $C_{\mathrm{R}} \leq 0.05$ and $C_{\phi} \leq 4^{\circ}$, respectively, where $C_{\mathrm{R}}=\left(R_{\mathrm{Max}}-R_{\mathrm{Min}}\right) /\left(R_{\mathrm{Max}}+R_{\mathrm{Min}}\right)$ and $C_{\phi}=\phi_{\mathrm{Max}}-\phi_{\text {Min }}$, with $R_{\text {Max }}, R_{\text {Min }}, \phi_{\text {Max }}$, and $\phi_{\text {Min }}$ being $\max /$ min values of relevant variables. These values are significantly lower than the theoretical maximum values of $C_{\mathrm{R}}=1$ and $C_{\phi}=180^{\circ}$ (discussion of the phase contrast will be presented in the Experimental Results section).

Figure 3 shows the simulated dependence of the amplitude and phase of the rf spectroscopy signal measured by the atomic magnetometer on the resultant magnetic field. Moving along rows/ columns of Fig. 3 is equivalent to a variation in the $\hat{y} / \hat{z}$ component of the resultant magnetic field. Firstly, we consider changes in the signal profile with $B_{z}^{\prime}$ for $B_{y}^{\prime}=0$, i.e., plots in the middle column of Fig. 3. Complete compensation of the primary field component in the resultant magnetic field monitored by the atomic magnetometer $\left(B_{y}^{\prime}=0, B_{z}^{\prime}=0\right)$ leads to an increase in amplitude and phase contrast $\left(C_{R}=0.44\right.$ and $C_{\phi}=124^{\circ}$, central plot in the third row of Fig. 3) with respect to previously quoted values but still does not produce maximum contrast values. The reason is that for $\left|\overrightarrow{B^{\prime}}\right|=0$, the signal amplitude becomes $R=\sqrt{b_{y}^{2}+b_{z}^{2}}$, where the high contrast component $b_{y}$ is reduced by the slowly varying offset of $b_{z}$ (Fig. 2). The phase of the rf spectroscopy signal $(\phi)$ changes by $180^{\circ}$, only when there is a change in the sign in the numerator and a singularity in the denominator. This indicates that the condition for achieving maximum contrast, with respect to the $\hat{z}$ axis components, is when $B_{z}^{\prime}+b_{z, \max }=0$. In the following, we refer to this condition, along with $B_{y}^{\prime}=0$, as the compensation point. Figure 3 confirms that the maximum amplitude and phase contrast are observed for $B_{z}^{\prime}+b_{z, \max }=0$, in each column, i.e., for an arbitrary value of $B_{y}^{\prime}$.

Moving right/left from the compensation point amounts to the addition of a negative/positive reference level. This induces a symmetry change of the profiles observed on either side of the compensation point. The amplitude and phase contrasts $\left(C_{R}=1\right.$, $\left.C_{\phi}=180^{\circ}\right)$ do not deteriorate in the vicinity of the compensation point as long as $\left|B_{y}^{\prime}\right| \leq b_{y \text {,max }}$. In the vicinity of the compensation point, modelling predicts phase jumps in the magnetic resonance signal by nearly $180^{\circ}$ over the recess area. The reason for the sudden phase change is the presence of a $\mathrm{z}$ component in the resultant $\mathrm{rf}$ field in the denominator of the arctan function that defines the phase of the rf spectroscopy signal.

\section{EXPERIMENTAL RESULTS}

We adapt as a test bed for the experimental exploration of $\mathrm{rf}$ magnetic field compensation, changes in the amplitude and phase of the rf spectroscopy signal recorded with an rf coil scanned across the defect in the form of recesses $(24.5 \mathrm{~mm}$ diameter, 2.4 $\mathrm{mm}$ deep) in a $6 \mathrm{~mm}$ thick plate of aluminium and carbon steel. ${ }^{17,18}$ We begin our studies from the realization of the compensation point. Experimentally, this is achieved by tuning the distance between the compensation coils and the vapour cell (Fig. 1). The coil located above the vapour cell is positioned on the axis with the 

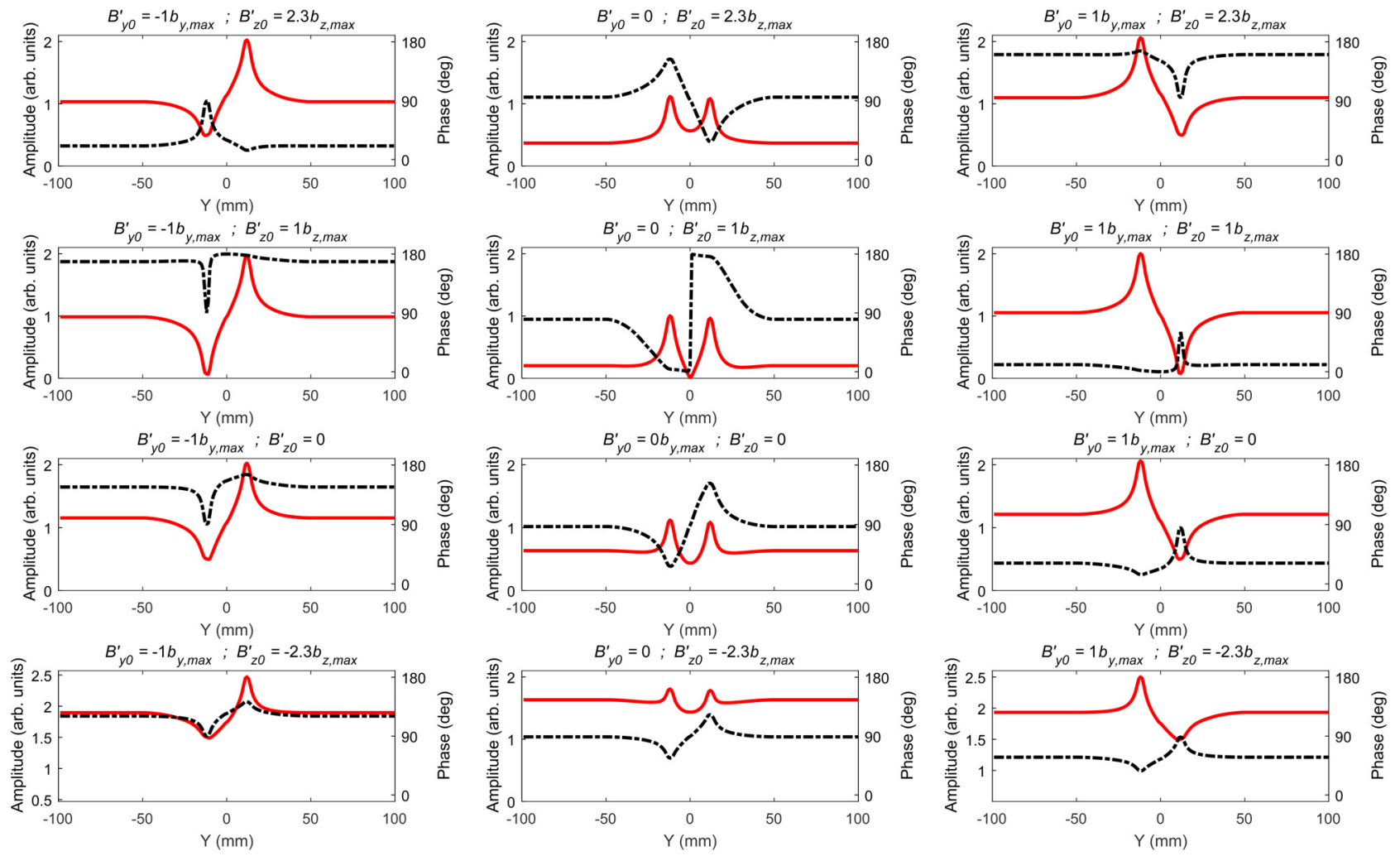

FIG. 3. The modelled change in the signal phase (dotted black line) and the amplitude (solid red line) of the magnetic resonance signal over a recess recorded by a magnetometer for various amplitudes of the primary field components. The vertical axis of the image array represents changes in the vertical component, while the horizontal axis represents changes in the horizontal component of the primary field. Amplitude is expressed in units of $b_{\text {y,max }}$.

rf coil producing the primary field. The optimum location of this coil along $\hat{z}$ is established by minimising the amplitude of the $\mathrm{rf}$ spectroscopy signal over the recess. The position of the other compensation coil can be adjusted in all three directions (only the $\hat{y}$ direction matters, the other two axes are effectively insensitive). The presence of this coil is particularly important in measurements with objects with a high magnetic permeability, where a misalignment of the rf coil with respect to the surface normal of the sample results in significant $B_{y}, B_{x}$ components of the primary field. Figure 3 indicates that compensation in the horizontal direction results in symmetric amplitude and phase profiles. This factor is utilised in searches for the compensation point.

Figure 4 shows the changes in (a) amplitude, $C_{R}$ (red diamonds), and (b) phase (black points), $C_{\phi}$, contrast as a function of the distance of the vertical coil from the compensation point along $\hat{z}$. The measurement was performed with an aluminium plate. Green squares in Fig. 4(a) represent the change of the rf spectroscopy signal amplitude measured in the centre of the recess. Both plots confirm the contrast is maximised at the compensation point. For reference, we show the amplitude/phase contrast value recorded without $\mathrm{rf}$ compensation fields [red/blue solid horizontal line in Figs. 4(a) and 4(b)]. A maximum phase contrast in Fig. 4(b) is less than the theoretical maximum value of $180^{\circ}$ since the primary field and magnetometer axes are not orthogonal. Consequently, the signal includes components other than $b_{y}$. The difference, $\sim 10^{\circ}$, is a measure of the systematic error (offset) of results presented.

The phase contrast $C_{\phi}$, as shown in Fig. 4(b), was calculated with an underlying assumption that requires brief explanation. If one follows the change of the field orientation while crossing the recess, a phase change of more than $180^{\circ}$ can be determined. From a practical perspective, the phase contrast should vary between $0^{\circ}$ and $180^{\circ}$, e.g., $C_{\phi}\left(\phi_{\text {Max }}-\phi_{\text {Min }}=0^{\circ}\right)=C_{\phi}\left(\phi_{\text {Max }}-\phi_{\text {Min }}=360^{\circ}\right)$. For a phase difference equivalent to a reflex angle, we determine the value of $C_{\phi}$ to be $360^{\circ}$ minus the recorded phase difference. As mentioned before, because of a misalignment, there is a systematic offset present in the data plotted in Fig. 4(b) and we took this offset into account when evaluating $C_{\phi}$ for reflex angles.

Figures 5(a)-5(c) and 5(g)-5(i) show the amplitude/phase images of a $64 \times 64 \mathrm{~mm}^{2}$ area of a $6 \mathrm{~mm}$ thick carbon steel plate containing a $24.5 \mathrm{~mm}$ diameter recess that is $2.4 \mathrm{~mm}$ deep recorded for three values of the horizontal component of the compensation rf field. The vertical cross-sections through the centre of the amplitude/phase images are shown in Figs. 5(d)-5(f) and 5(j)-5(1). They are equivalent to the calculated amplitude profiles from the second row of Fig. 3. The cases illustrated in Figs. 5(b) and 5(e) 

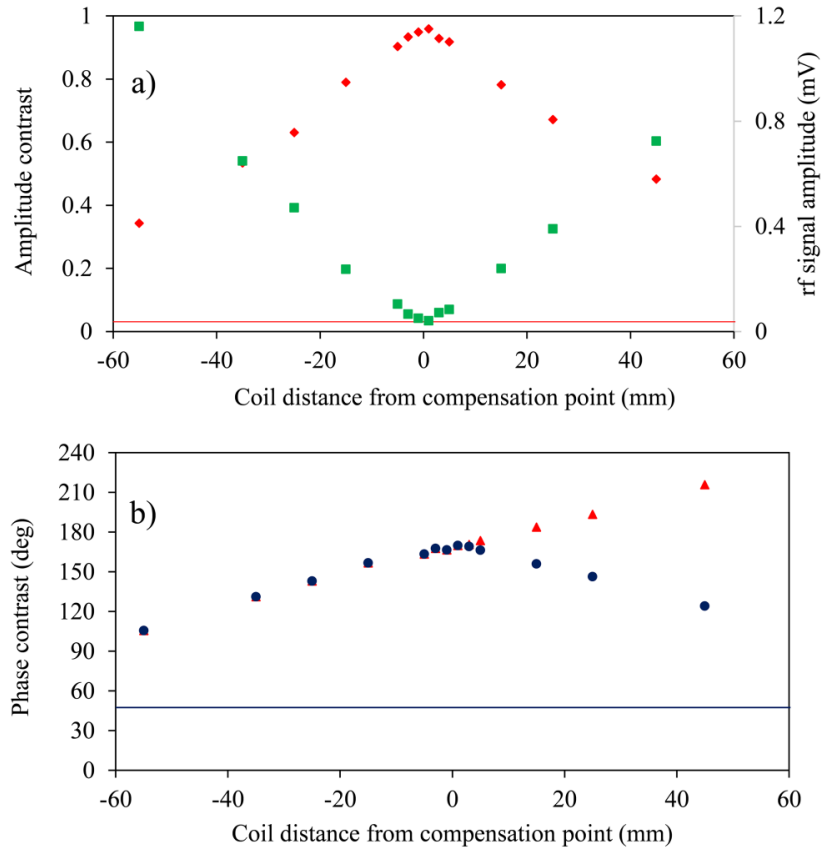

FIG. 4. The (a) amplitude (red diamonds) and (b) phase (blue dots) contrast measured for different locations of the compensation coil above the vapour cell from the compensation point. Green squares in (a) represent the change of the If spectroscopy signal amplitude. (a) Solid red/(b) blue line shows the amplitude/ phase contrast in the absence of a compensation field. Red triangles in (b) show the unadjusted value of $\phi_{\mathrm{Max}}-\phi_{\mathrm{Min}}$

and $5(\mathrm{~h})$ and $5(\mathrm{k})$ represent the compensation point. Near the compensation point, the amplitude image produced by a recess is symmetrical, i.e., two maxima corresponding to rising and falling edges. This results from zeroing the primary field that sets the background reference for changes in the secondary field generated by the magnetisation $\left(R \sim\left|b_{y}\right|\right)$. The bright maximum in Fig. 5(b) is surrounded by a dark ring; in other words, the profiles created by the two recess edges are not perfectly symmetrical. This might be due to imperfect compensation of the static magnetic field, in addition to the rf fields. Imperfect compensation of the static magnetic field, i.e., a variation of the ambient magnetic field, is equivalent to a change in the direction of the atomic magnetometer axis, which could affect the observed image [see the following section, Figs. 7(b) and 7(c)]. This could be minimised by increasing the operating frequency. The magnetic response of the sample can be thought of in terms of changes in the rf coil inductance. Inhomogeneity in the magnetisation across the plate would be seen as a variation of the primary field strength, meaning rf field compensation is valid only locally. Change of the rf field strength monitored by the magnetometer can be seen by the variation in the background level. There is a change in the shape and symmetry of the amplitude profiles on either side of the compensation point, related to the passage from $R=\left|b_{y}+B_{y}^{\prime}\right|$ [Fig. 5(d)] through $R \sim\left|b_{y}\right|$ [Fig. 5(e)] to $R=\left|b_{y}-B_{y}^{\prime}\right|$ [Fig. 5(f)]. The phase contrast decreases as we move away from the compensation point
[Figs. 5(g)-5(i)]. Importantly, the area where the phase departs from its background value decreases away from the compensation point. This might have practical consequences in a measurement with a coarse spatial step, since the phase change generated by the recess could be non-visible as it would be equivalent to spatial integration of the phase change over a larger area.

The benefit of rf compensation can be demonstrated in the experiment with an increased lift-off distance, $6 \mathrm{~mm}-7 \mathrm{~mm}$. The starting point for this is the measurement of the phase contrast in the standard configuration (the rf coil producing the primary field located $1 \mathrm{~mm}-2 \mathrm{~mm}$ above the sample surface, no rf compensation). The phase contrast for a case of a $12 \mathrm{~mm}$ diameter recess that is $2.4 \mathrm{~mm}$ deep in a $6 \mathrm{~mm}$ thick aluminium plate is $C_{\phi}=40^{\circ}$. An increase of the lift-off $(6 \mathrm{~mm}-7 \mathrm{~mm})$ results in a reduction of the strength of the primary field at the sample and, consequently, the secondary field. At the same time, for a fixed distance between the vapour cell and the sample (Fig. 1), the primary field component monitored by the atomic magnetometer increases. This causes a reduction of the recorded phase contrast to $C_{\phi}=20^{\circ}$. With the addition of the compensation rf field, we were not only able to recover the initial phase contrast value but even increase it to its maximum value, $C_{\phi}=180^{\circ}$.

The atomic magnetometer evaluates the oscillating magnetic field strength through the measurement of the atomic Zeeman coherence amplitude produced by this field in the atomic vapour polarized along the direction of the static magnetic field, $\vec{B}_{\text {bias }}{ }^{21}$ Since only magnetic fields oscillating orthogonally to the $\vec{B}_{\text {bias }}$ direction can generate atomic coherences, the magnetometer is insensitive to rf fields along $\vec{B}_{\text {bias }}$. For $\vec{B}_{\text {bias }}$ aligned along $\hat{z}$, this property of the atomic magnetometer is equivalent to the first part of the compensation condition, $B_{z}^{\prime}+b_{\mathrm{z}, \max }=0$, i.e., the absence of the $\mathrm{z}$-component of the resultant $\mathrm{rf}$ field in the magnetometer signal. Figure 6 shows the modified measurement setup used in the demonstration of this type of compensation. The static bias magnetic field is directed along $\hat{z}$ and set to the same strength used in previous measurements (equivalent to the Larmor frequency about $12.6 \mathrm{kHz}$ ). The pump laser beam is aligned along the bias magnetic field. Instrumentation includes a set of rf coils compensating the horizontal components of the primary field. The position of these compensating coils is varied such that the rf spectroscopy signal is minimised without the sample, in other words, to compensate the horizontal components of the primary field $\left(B_{x}^{\prime}=0\right.$ and $\left.B_{y}^{\prime}=0\right)$. In this modified configuration, the amplitude $\left[R=\sqrt{\left(b_{x}\right)^{2}+\left(b_{y}\right)^{2}}\right]$ and the phase $\left(\phi=\arctan \frac{b_{x}}{b_{y}}\right)$ of the rf spectroscopy signal reflects variations in the amplitude and phase of the horizontal components of the secondary field.

Figure 7 illustrates the benefits of, and differences between, the two compensation schemes discussed. It shows the images of a $64 \times 64 \mathrm{~mm}^{2}$ area of a $6 \mathrm{~mm}$ thick aluminium plate containing a $24 \mathrm{~mm}$ diameter recess that is $2.4 \mathrm{~mm}$ deep recorded in three different configurations: (a) without compensation, (b) with compensation performed with two rf coils, and (c) with a rotated bias magnetic field and compensation coils. The images represent the change of the amplitude of the rf spectroscopy signal. As mentioned before, for the uncompensated case, (a) the recorded profile 
(a)

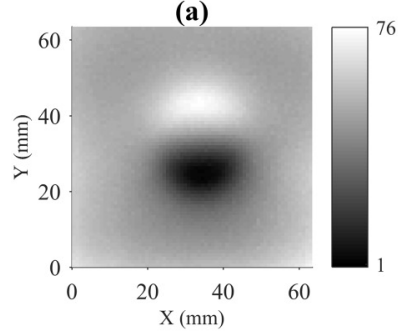

(b)

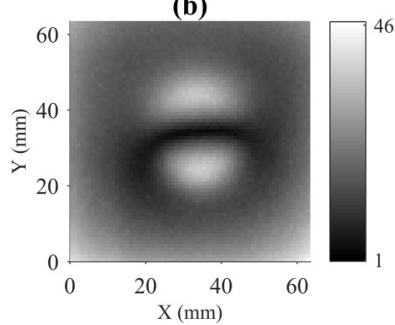

(c)

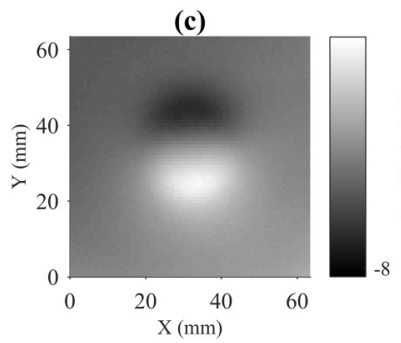

(d)

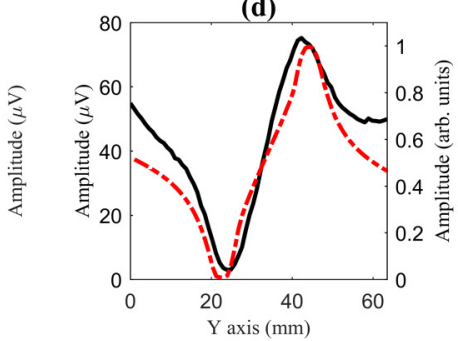

(e)

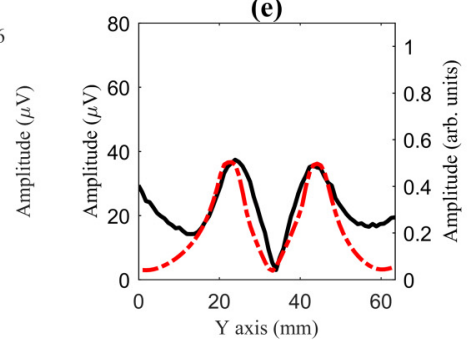

(f)

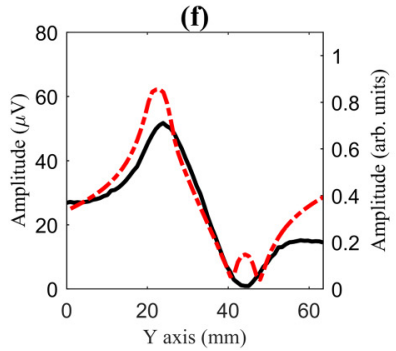

(g)

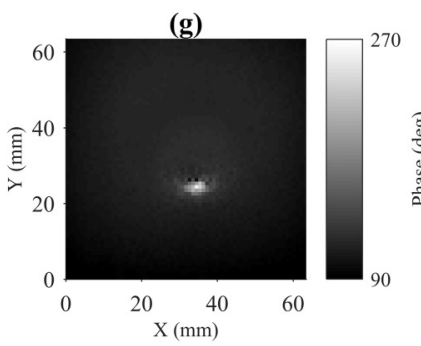

(h)

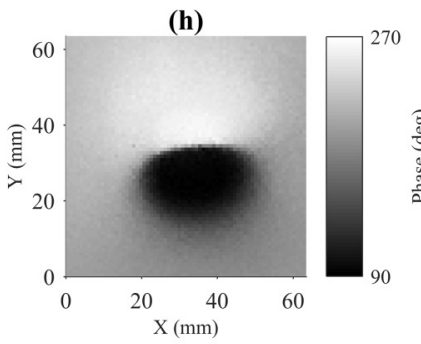

(i)

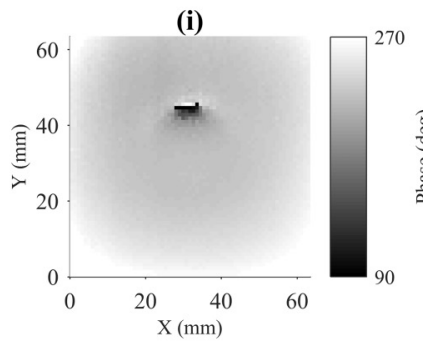

(j)

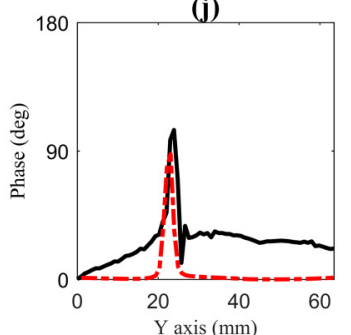

(k)

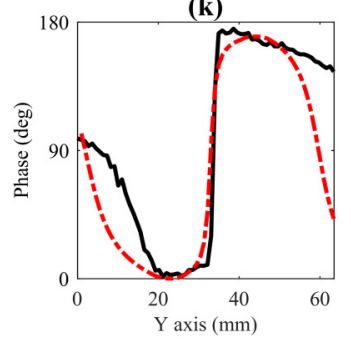

(l)

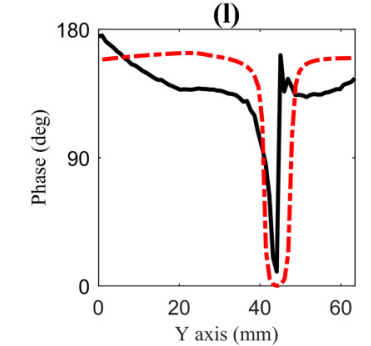

FIG. 5. (a)-(c) and (g)-(i) The measured change of the amplitude/phase of the rf spectroscopy signal (solid black line) over a $64 \times 64 \mathrm{~mm}^{2}$ area of a $6 \mathrm{~mm}$ thick carbon steel plate, containing a $24 \mathrm{~mm}$ diameter recess that is $2.4 \mathrm{~mm}$ deep, recorded for three values of the horizontal component of the compensation rf field around the compensation point. (d)-(f) and (j)-(l) The vertical cross-sections through the centre of the amplitude/phases images. The images have been recorded at $114.2 \mathrm{kHz}$. Dashed red line represents modelled amplitude and phase changes. The discrepancy between measured and modelled amplitude profiles, particularly in (e), comes from the edges of the plate.

shows a variation of the vertical component of the secondary field. In the compensated cases, the images show the $\hat{x}$ horizontal component (b) and both horizontal components (c) of the secondary field. The difference in the symmetry of the images results from the change of the direction of the bias field. In the case shown in Fig. 7 (b), $\vec{B}_{\text {bias }}$ is directed along the $\hat{x}$ axis and, therefore, only signatures produced by the recess edges parallel to that direction are present in the recorded profile (in other words, the edge parallel to $\vec{B}_{\text {bias }}$ produces an oscillating secondary field perpendicular to $\vec{B}_{\text {bias }}$ that can be seen by the magnetometer). In the case shown in Fig. $7(\mathrm{c}), \vec{B}_{\text {bias }}$ is directed along the $\hat{z}$ axis and the recorded profile shows the whole contour of the recess. We have calculated the amplitude contrast, $C_{R}$, as defined previously for the three images. The numbers confirm [(a) $C_{R}=0.04$; (b) $C_{R}=0.77$; (c) $\left.C_{R}=0.79\right]$ that implementation of the compensation schemes allows easier identification of the structural defects in the amplitude images. In the ideal case of the rf coil perpendicular to the surface of the sample, optimum compensation (i.e., defined by the

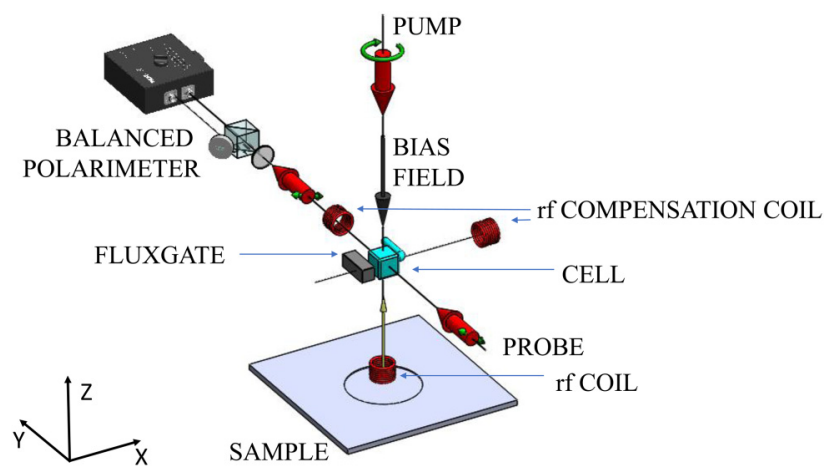

FIG. 6. Modified experimental setup with $\vec{B}_{\text {bias }}$ along $\hat{z}$. The pump laser beam orients the atomic vapour along the direction of the bias field. The horizontal components of the primary field are compensated in the vapour cell by a set of two rf coils oriented along the $\hat{x}$ and $\hat{y}$ directions. 
(a)

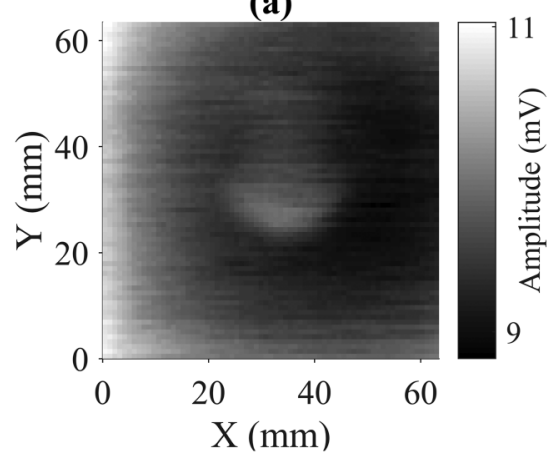

(b)

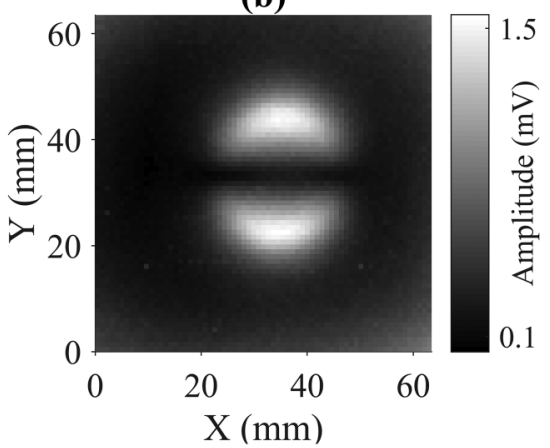

(c)

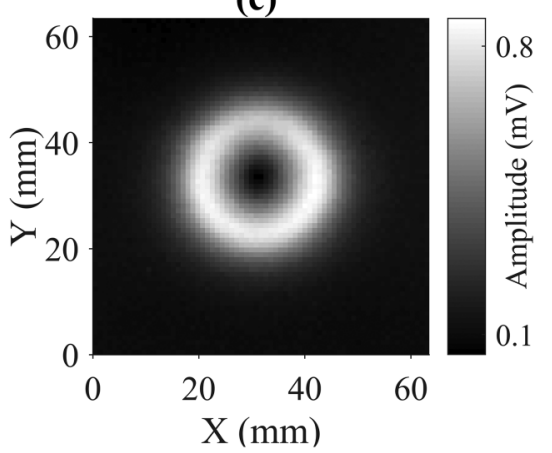

FIG. 7. The measured change of the amplitude of the rf spectroscopy signal over a $64 \times 64 \mathrm{~mm}^{2}$ area of a $6 \mathrm{~mm}$ thick aluminum plate, containing a 24 mm diameter recess that is $2.4 \mathrm{~mm}$ deep, recorded with three measurement configurations: (a) without rf field compensation, (b) with compensation performed with two rf coils (Fig. 1), and (c) with a rotated bias magnetic field and compensation coils (Fig. 6).

compensation condition) could be achieved only with the proper geometry of the atomic magnetometer (i.e., without compensation coils). In the case of the rf coil tilted with respect to the surface normal, compensation with only the atomic magnetometer aligned along the primary field direction would result in mixing of the secondary field components. This will be manifested by asymmetries in the Fig. 7(c) image. In this case, the optimum compensation could be achieved by aligning the atomic magnetometer's insensitive axis orthogonal to the sample surface and implementation of compensation coils in the horizontal plane.

As mentioned before, alignment of the bias magnetic field axis along the direction of the primary field ensures that the atomic magnetometer is immune to $B_{z}$. This is important in the context of possible saturation of the atomic magnetometer response by the $\mathrm{rf}$ fields. ${ }^{22}$ The rf field saturation amplitude at $30 \mathrm{kHz}(\sim 0.71 \mathrm{nT})$ is significantly smaller than the primary field amplitude $(\sim 74 \mu \mathrm{T}$, at the coil in the absence of the plate). In the current configuration, the $\mathrm{rf}$ coil and the sample are placed approximately $30 \mathrm{~cm}$ from the sensor. While the measured secondary field amplitudes $(\sim 16 \mathrm{pT}$ in carbon steel and $\sim 7 \mathrm{pT}$ in aluminium) are well within the sensitivity range of the magnetometer $\left(\sim 50 \mathrm{fT} / \mathrm{Hz}^{1 / 2},{ }^{17}\right)$, our measurement configuration provides the option of bringing the sample and the rf coil closer to the detector increasing the measured signal.

The amplitude and phase images recorded in the selfcompensation configuration, i.e., with the bias magnetic field parallel to the axis of the primary field, illustrates the semi-vector character of the secondary field measurement with an rf atomic magnetometer. Figure 8 shows the measured change of the amplitude, Fig. 8(a), and phase, Fig. 8(b), of the rf spectroscopy signal over the recess area. The measurement has been performed with a $1 \mathrm{~mm}$ diameter rf coil, which improves the spatial resolution of the image compared to that presented in Fig. 7(c). As mentioned before, the amplitude of the rf spectroscopy signal $\left[R=\sqrt{\left(b_{x}\right)^{2}+\left(b_{y}\right)^{2}}\right]$ reproduces the (a)

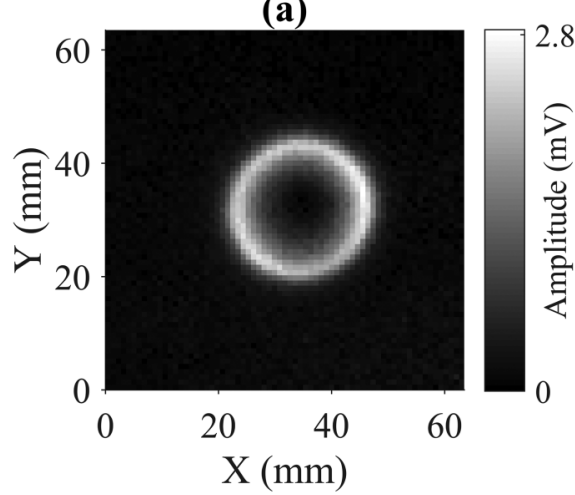

(b)

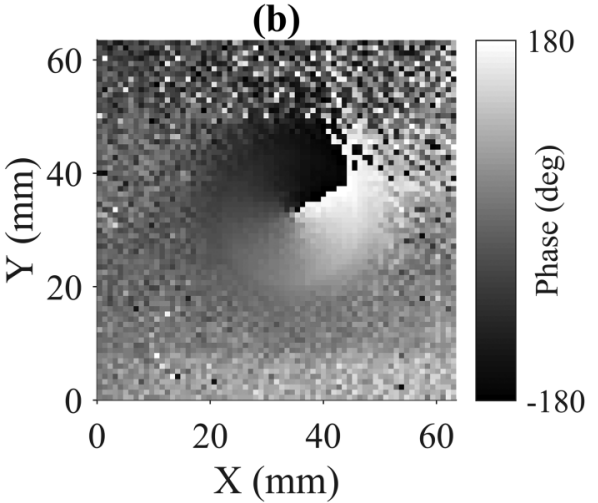

(c)

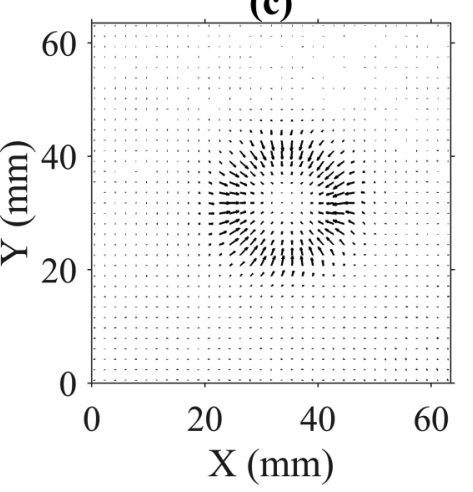

FIG. 8. The measured change of the amplitude (a) and phase (b) of the rf spectroscopy signal recorded in the self-compensation configuration (Fig. 6) at $30 \mathrm{kHz}$. The experimental conditions are the same as those for Fig. 7 (c) apart from the rf coil diameter, now $1 \mathrm{~mm}$, which improves the spatial resolution of the measurement. The amplitude and the phase images are used to re-create the map of the horizontal components of the secondary field (c). 
amplitude of the horizontal components of the secondary field. The phase change of the rf spectroscopy signal in Fig. 8(b) shows a vortex centred on the recess, which reflects the opposite direction of these components at opposite edges of the recess. While the phase image only provides information of the relative phase change, by arbitrarily defining the phase value at a single point, it is possible to re-create a map of the horizontal components of the secondary field [Fig. 8(c)]. The amplitude/phase of the secondary field is represented in Fig. 8 (c) by the length/orientation of the vector.

\section{CONCLUSIONS}

We have demonstrated two simple methods for improvement of the contrast in the images representing the variations of the amplitude and phase of the rf spectroscopy signal recorded by the atomic magnetometer in the inductive coupling non-destructive testing measurement. The methods are based on the compensation of the components of the resultant rf magnetic field monitored by the atomic magnetometer by either a set of coils or the geometry of the measurement. Reduction of the amplitude of the rf signal monitored by the atomic magnetometer through the compensation process does not compromise the ability for defect detection. On the contrary, monitoring of the signal phase in the compensated configuration provides the option of a clear $\left(180^{\circ}\right.$ phase change) signature of the inhomogeneity.

\section{ACKNOWLEDGMENTS}

P.B. was supported by the Engineering and Physical Sciences Research Council (EPSRC) (No. EP/P51066X/1).

\section{REFERENCES}

${ }^{1}$ L. Ma and M. Soleimani, "Magnetic induction tomography methods and applications: A review," Meas. Sci. Technol. 28, 072001-072012 (2017).

${ }^{2}$ H. Griffiths, "Magnetic induction tomography," Meas. Sci. Technol. 12, 1126-1131 (2001).

${ }^{3}$ B. A. Auld and J. C. Moulder, "Review of advances in quantitative eddy current nondestructive evaluation,” J. Nondestr. Eval. 18, 3-36 (1999).

${ }^{4}$ L. Perez, J. Le Hir, C. Dolabdjian, and L. Butin, "Investigation in detection of fatigue cracks under rivet head airframe using improved GMR magnetometer in an eddy current system," J. Electr. Eng. 55, 73-76 (2004); available at http://iris. elf.stuba.sk/JEEEC/data/pdf/10s_104-20.pdf

${ }^{5}$ A. Sophian, G. Tian, and M. Fan, "Pulsed eddy current non-destructive testing and evaluation: A review," Chin. J. Mech. Eng. 30, 500 (2017).
${ }^{6}$ T. Dogaru and S. T. Smith, "Edge crack detection using a giant magnetoresistance based eddy current sensor," Nondestr. Test. Eval. 16, 31-53 (2000).

${ }^{7_{T}}$. Dogaru and S. T. Smith, "Giant magnetoresistance-based eddy-current sensor,” IEEE Trans. Magn. 37(5), 3831-3838 (2001).

${ }^{8}$ P. Ripka and M. Janosek, “Advances in magnetic field sensors," IEEE Sens. J. 10, 1108-1116 (2010).

${ }^{9}$ H. J. Krause and M. V. Kreutzbruck, "Recent developments in SQUID NDE," Physica C 368, 70-79 (2002).

${ }^{10}$ J. Storm, P. Hömmen, D. Drung, and R. Körber, "An ultra-sensitive and wideband magnetometer based on a superconducting quantum interference device," Appl. Phys. Lett. 110, 072603 (2017).

${ }^{11}$ A. Wickenbrock, S. Jurgilas, A. Dow, L. Marmugi, and F. Renzoni, "Magnetic induction tomography using an all-optical $87 \mathrm{Rb}$ atomic magnetometer," Opt. Lett. 39, 6367-6370 (2014).

${ }^{12}$ C. Deans, L. Marmugi, S. Hussain, and F. Renzoni, "Electromagnetic induction imaging with a radio-frequency atomic magnetometer," Appl. Phys. Lett. 108, 103503 (2016).

${ }^{13}$ A. Wickenbrock, N. Leefer, J. W. Blanchard, and D. Budker, "Eddy current imaging with an atomic radio-frequency magnetometer," Appl. Phys. Lett. 108, 183507 (2016).

${ }^{14}$ C. Deans, L. Marmugi, and F. Renzoni, "Through-barrier electromagnetic imaging with an atomic magnetometer," Opt. Express 25, 17911-17917 (2017).

${ }^{15}$ I. M. Savukov, S. J. Seltzer, and M. V. Romalis, "Detection of NMR signals with a radio-frequency atomic magnetometer," J. Magn. Res. 185, 214 (2007).

${ }^{16}$ I. M. Savukov, S. J. Seltzer, M. V. Romalis, and K. L. Sauer, "Tunable atomic magnetometer for detection of radio-frequency magnetic fields," Phys. Rev. Lett. 95, 63004 (2005).

${ }^{17} \mathrm{P}$. Bevington, R. Gartman, and W. Chalupczak, "Imaging of material defects with a radio-frequency atomic magnetometer," Rev. Sci. Instrum. 90, 013103 (2019).

${ }^{18}$ P. Bevington, R. Gartman, W. Chalupczak, C. Deans, L. Marmugi, and F. Renzoni, "Non-destructive structural imaging of steelwork with atomic magnetometers," Appl. Phys. Lett. 113, 063503 (2018).

${ }^{19}$ W. Chalupczak, R. M. Godun, S. Pustelny, and W. Gawlik, "Room temperature femtotesla radio-frequency atomic magnetometer," Appl. Phys. Lett. 100, 242401 (2012).

${ }^{20}$ G. Bevilacqua, V. Biancalana, P. Chesssa, and Y. Dancheva, "Multichannel optical atomic magnetometer operating in unshielded environment," Appl. Phys. B 122, 103 (2016).

${ }^{21}$ W. Chalupczak, R. M. Godun, and S. Pustelny, "Radio-frequency spectroscopy as a tool for studying coherent spin dynamics and for application to radio-frequency magnetometry," Adv. At. Mol. Opt. Phys. 67, 297-336 (2018).

${ }^{22}$ T. Zigdon, A. D. Wilson-Gordon, S. Guttikonda, E. J. Bahr, O. Neitzke, S. M. Rochester, and D. Budker, "Nonlinear magneto-optical rotation in the presence of a radio-frequency field," Opt. Express 18, 25494 (2010). 